\title{
Evaluation of error in temperature starting from the Slit Response function and calibration curve of a thermal focal plane array camera
}

Olivier RIOU, Jean Félix DURASTANTI, Vincent TORTEL

Centre de recherches en Thermique. Environnement et Système - CERTES

IUT De Sénart. Avenue Pierre Point 77127 LIEUSAINT

\section{Summary}

The sector of the thermographic control of the electric installations is a large user of thermal cameras. This sector has a very large variety of cameras of which image quality is an asset. However, it is not enough to guarantee an exact measurement of temperature. In this work, we give an advanced reading of the Slit response function. It enables us to quantify the systematic error in temperature for a wire-shape object at the $T_{1}$ temperature for any wire cross dimension $d$ and back side radiometric temperature $\left(T_{0}\right)$, relative to target-camera distance $D$ and using a thermal focal plane array camera. The procedure is detailed and a result obtained on a commercial camera is shown.

\section{Introduction}

For thermal focal plane camera, we can classify the characteristics of the thermal cameras in two distinct application fields [1] : imagery (the camera provides images) and measurement (the camera is a radiometric apparatus). This is why French standart treats about two resolutions: the Spatial Resolution of Observation or PRSO and the Spatial Resolution of Measurement or PRSM.

A fine analysis of the process of formation of the images shows the limits for spatial resolution. A study of the spot of diffusion indicates a very strong sensitivity with the the optical number $\mathrm{N}$ and the camera's spectral band. Applied to the focal plane array (FPA) cameras. the spot of diffusion is associated with the impulse response $D(x)$ of a detector. In this formalism, we will then be able to give an interpretation of the signal provided by the observation of a slit presenting a strong thermal contrast (Slit Response Function).

Such test will be detailed in connection with the exactitude in temperature. One will point out the characterization of spot size ratio (SSR) very useful in pyrometry but new in thermography.

In this article, one will call upon the French standards of thermography [2]. Nevertheless FPA cameras being posterior to the standards, those became incomplete and sometimes unsatisfactory.

The metrological aspects will be treated in the respect of the International Vocabulary from Metrology or VIM [3].

\section{Spatial resolution of observation}

In imagery field, it is of use to define the Spatial Resolution of Observation (PRSO) by the number of detectors whereas the standard specifies it by an angle. Thus, the PRSO is the product of the number of detectors on a line (ndh) and number of detectors on a column $(n d V)$ : for example $n d H \times n d V=320 \times 240$. 
Because of matrices of non standardized size, one does indicate an objective by his angle FOV - Field Of View - under which the camera sees the thermal scene (HFOV: horizontal angle, and VFOV: vertical angle). In geometrical optics, a detector of the FPA sees an elementary surface $\Delta S$ of the thermal scene under an angle called IFOV (Instantaneous Field Of View) (see fig. 1).

There are

$$
\operatorname{IFOV}\left({ }^{\circ}\right)=\operatorname{HFOV}\left({ }^{\circ}\right) / \mathrm{ndH}=\operatorname{VFOV}\left({ }^{\circ}\right) / \mathrm{ndV}
$$

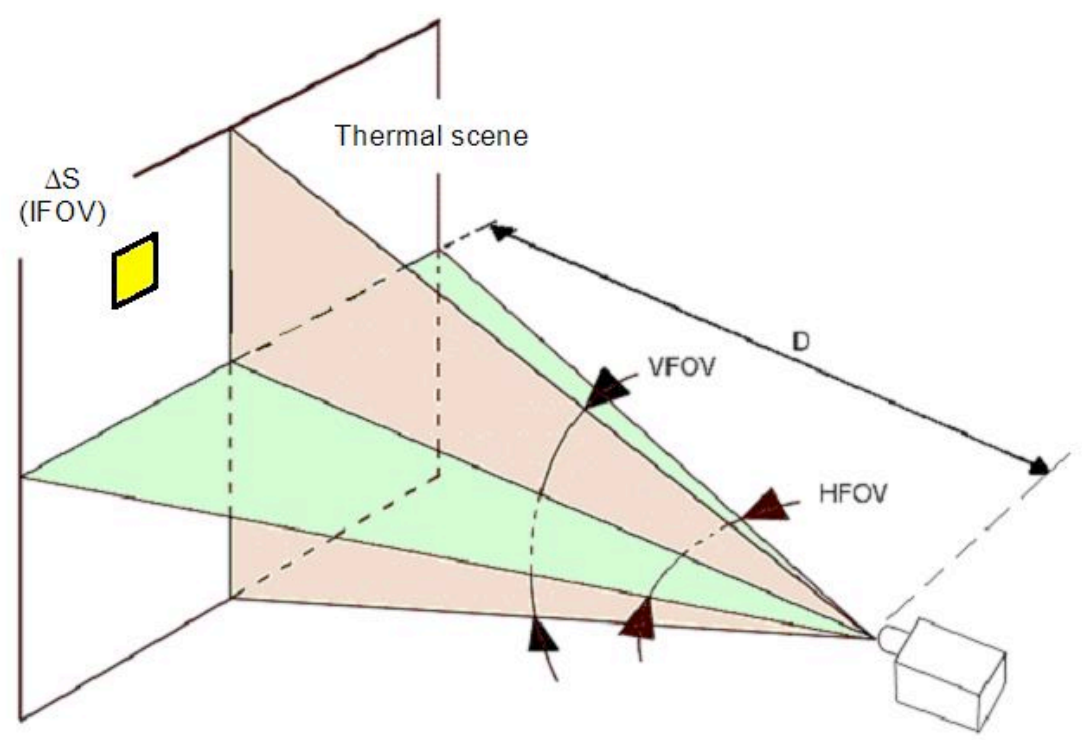

Fig. 1. angles HFOV. VFOV and IFOV ( figure is issue from reference [4])

For practical reasons. the FOV is expressed in degrees of angle $\left(^{\circ}\right)$ and the IFOV in milliradians (mrad) or millimetres with the distance from measurement $D(\mathrm{~mm}$ @ D). The values of the IFOV in mrad or $\mathrm{mm} @ 1 \mathrm{~m}$ are identical.

The PRSO of a camera having an objective of HFOV $=18^{\circ}$ and a matrix of $160 \times 120$ detectors can also be expressed by the value of the IFOV $\approx 2 \mathrm{mrad}$ or also $1.2 \mathrm{~mm} @ 0.6 \mathrm{~m}$ where $0.6 \mathrm{~m}$ is the minimal distance from focusing of the objective.

\section{Limits of the spatial resolution of observation in focal plane array camera}

The nature of the light imposes a limit on the spatial resolution of observation. Mainly because of the phenomena of diffraction and aberration, the image of a point is not a point. These phenomena degrade the spatial resolution of observation of any optical systems.

Diffraction and the geometric aberrations are physical phenomena present in systems using pupils (lens. diaphragm...). This phenomena transform the image of a point into a spot of diffusion.

The diffusion spot size will depend mainly on the optical number $\mathrm{N}$ seen in $\mathrm{f} / \mathrm{N}$ of the optical system. Sometimes antagonistic, the deformations could be corrected. The processes of corrections are well-known by opticians. In some simple cases, it is possible to determine the angular extent of the spot of diffusion. 
In table 1, we evaluate the diffusion spot size of diffusion in the case of a thin Germanium lens used for spectral band LWIR $(n=4, v=1112)$. The lens presents a focal distance of $100 \mathrm{~mm}$ and a diameter $2 \mathrm{~h}=50 \mathrm{~mm}$. Evaluations are given to FOV of $20^{\circ}$ and optic number $\mathrm{N}=\mathrm{f} / 2 \mathrm{~h}=2$ ( $\mathrm{f} / 2$ optic).

Table 1.Diffusion spot size of thin germanium lens

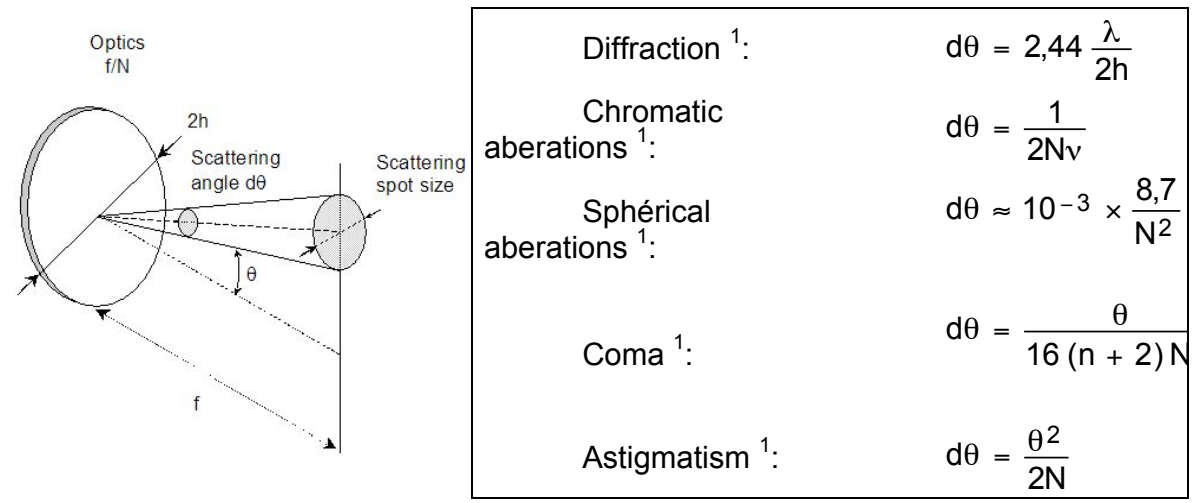

${ }^{1}$ See Reference 5

\begin{tabular}{|c|c|c|}
\hline & Scatering angle & Scatering spot \\
\hline Diffraction: & 0.488 & 48,8 \\
\hline Chromatism: & 0,225 & 22,5 \\
\hline Spherical aberration: & 1,088 & 108,8 \\
\hline Coma: & 1,364 & 136,4 \\
\hline Astigmatism: & 7,615 & 761,5 \\
\hline Scattering angle exept & $1,8 \mathrm{mrad}$ & $180 \mu \mathrm{m}$ \\
\hline coma and astigmatism: & $1,6 \mathrm{mrad}$ & $160 \mu \mathrm{m}$ \\
\hline Achromatic optics: & &
\end{tabular}

This example shows that PRSO is strongly limited by the quality of used lens. specially at the edge of the images where coma an astigmatisme are dominant.

At the center of image and for an achromatic optics, the scattering angle cannot be less than 1.6 mrad for an optic number of $f / 2$ in the LWIR band. It is not rare to meet cameras of optic number greater ( $f / 1)$. For such cameras, The scattering angle is strongly increased.

Other parameters of design limit the spatial resolution of observation: let us quote the variation of its local sensitivity of the detector. the electronics of reading. etc...

All these factors give the impulse linear response $D(x)$ of the system. One models the impulse response by a Gaussian function in which $\sigma$ represents a caracteristic scattering diffusion corresponding to $84 \%$ of received flow $( \pm \sigma)$. the FPA camera are conceived in such manner so $\sigma$ is of the same order as the dectector dimension. The associated solid angle $\sigma / f$ (f: focal distance) is identified with the instantaneous field of view (IFOV). 


\section{Slit Response Function (SRF)}

In the impulse response formalism. it is possible to determine the spatial resolution criteria of the system. The relative maximum value of the signal given by SRF can be calculated by numerical integration starting from the impulse response linear $\mathrm{D}(\mathrm{x})$

$$
L_{\max }(d)=\frac{\int_{-d / 2}^{d / 2} e^{-\frac{x^{2}}{\sigma^{2}}} d x}{\int_{-\infty}^{\infty} e^{-\frac{x^{2}}{\sigma^{2}}} d x}
$$

SRF is then very useful to evaluate how many flux it is possible to measure according to $d / \sigma$. Some particular values are presented in table 2 .

Table 2. Evaluation of the relative maximum value of the signal given by a slit

\begin{tabular}{|l|l|l|l|l|}
\hline$L_{\max }(d)$ & 0.5 & 0.8 & 0.9 & 0.99 \\
\hline$d / \sigma$ & 0.96 & 1.8 & 2.32 & 3.64 \\
\hline
\end{tabular}

The value corresponding to $50 \%$ of SRF is represented by dimension $\sigma$ or the angular $\sigma / f \approx I F O V$ of the camera.

SRF test is very useful when one search to clarify the precautions to be taken for "correct" temperature measurement of a wire. To carry out the test. we usually use a slit: The lips of the slit present the radiometric temperature $T_{0}$ ( back side radiometric temperature) associated to the camera level (or thermosignal) $\mathrm{L}_{0}$. The body behind the slit. is at the radiometric temperature $T_{1}$ ( slit radiometric temperature) associated to the thermosignal $L_{1}$ higher than $L_{0}$ ( see figure 2 )

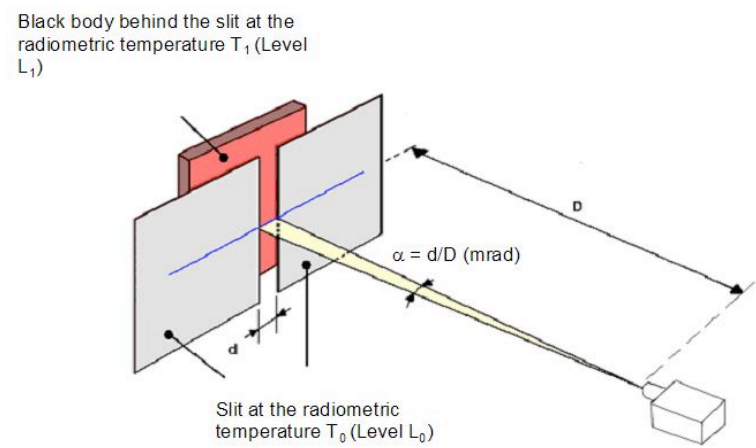

Fig. 2a. Slit Response Function. Camera sees slit lips of radiometric temperature $T_{0}$ (back side radiometric temperature) and The body behind the slit of radiometric temperature $T_{1}$ ("slit "temperature). Slit width is $d$ and $D$ is the distance slit-camera (Figure is issue from reference 4) 


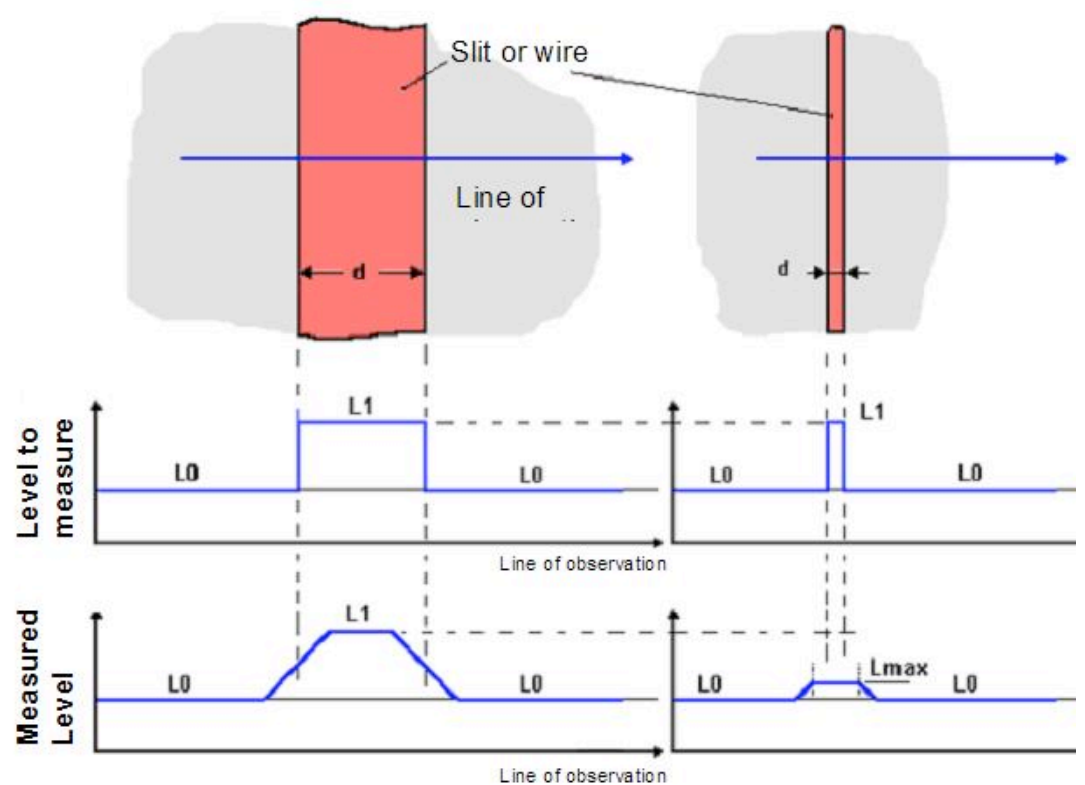

Fig. $2 b$. Slit Response Function. Below a certain value of $d$,. the $L_{\max }$ value of the thermosignal decrease from $L_{1}$ to $L_{0}$ (figure is issue from reference 4 )

The normalized response of the camera is then

$$
\text { SRF }=\frac{L_{\max }-L_{0}}{L_{1}-L_{0}}
$$

d can be expressed in length $(\mathrm{mm})$ or angle for the distance from measurement $D \alpha=d / D$ (mrad) or Distance to Size Ratio $D / d$.

\section{Exactitude in temperature}

When we speak about measurement. the system must show the general characteristics of a measuring instrument: repeatability and exactitude. To quantify exactitude in temperature. it is necessary to give to the object an angular dimension which makes the SRF within interval $[1,1-\varepsilon]$ ( $\varepsilon$ is the error in measurement). A variation of $\varepsilon \%$ on the relative value of the signal corresponds to a systematic error in temperature given by the relation:

$$
\Delta \mathrm{T}=\frac{\Delta \mathrm{N}}{\langle\mathrm{S}\rangle}
$$

where $\Delta \mathrm{N}$ is the variation in level on the SRF and $\left\langle\mathrm{S}>\right.$ in Level $/{ }^{\circ} \mathrm{C}$ is the sensitivity of the camera on the interval $\left[T_{0}, T_{1}\right]$. Systematic error $\Delta T$ is generally negative because the apparent temperature is estimated by defect when $T_{1}>T_{0}$. The sensitivity is deduced from the calibration curve of the camera.

Systematic error $\Delta \mathrm{T}$ is then dependant to back side radiometric temperature $T_{0}$, slit temperature $T_{1}$ and Distance to size ratio $D / d$. 


\section{Evolution of the test}

With some exceptions, the cameras are linear in measurement of flux: they provide a thermosignal proportional to the flux to be measured; this means that the FRF remains unchanged whatever are the slit lips and slit temperatures. One can deduce the results in level for any back side radiometric temperature increasing from $\mathrm{T}_{0}$ to $\mathrm{T}_{1}$. Naturally. the study also supposes that the behavior of the camera remains homothetic on the usual distances of measurements. In this context, we propose a second reading of the SRF. The approach is as follows:

We measure the SRF for the slit lips temperature $T_{0}$ (for example $20^{\circ} \mathrm{C}$ ) and slit temperature $\mathrm{T}_{1}$ (for example $70^{\circ} \mathrm{C}$ ). The normalized FRF of the camera is calculated for each d by FRF $=\left(L_{\max }-L_{0}\right) /\left(L_{1}-\right.$ $\left.L_{0}\right)$ and the result is expressed in radiometric levels in $\%$ of the maximal level. Levels are extracted from temperature via the camera's calibration curve. A function test of the normalized SRF is then determined by the method of least squares.

This function test enables us to calculate the variation in temperature for a slit at $70^{\circ} \mathrm{C}$ for different bottom temperature varying between $20^{\circ} \mathrm{C}$ and $70^{\circ} \mathrm{C}$ by step of $5^{\circ} \mathrm{C}$. The result is associated to the distance to size ratio $D / d$.

we treat finally the result by classifying $D / d$ which guarantee a maximum systematic error lower than -1 . $-2 .-5 .-10 .-15$ or $-20^{\circ} \mathrm{C}$.

This procedure was applied to a camera (IFOV $2.1 \mathrm{mrad}$ ). results are showed on figure 3 .

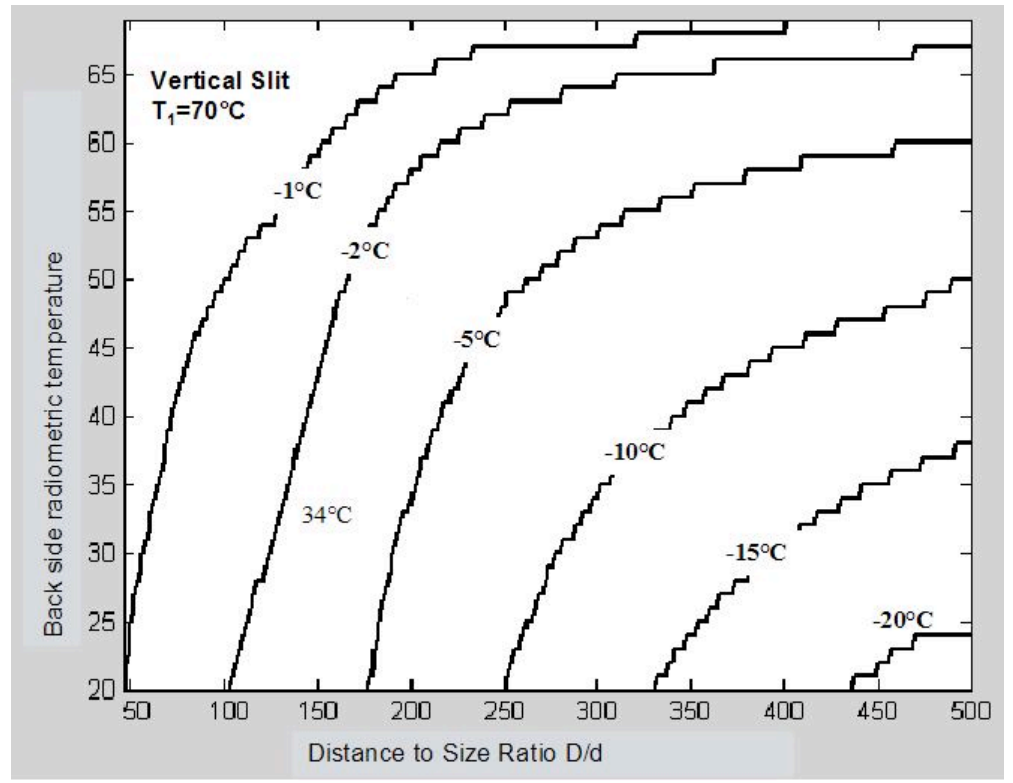

Fig. 3. Error systematic data for a vertical wire with $70^{\circ} \mathrm{C}$ in a content radiometric varying between 20 and $70^{\circ} \mathrm{C}$ according to distance to Size ratio $\mathrm{D} / \mathrm{d}$

How to read this graph? Let us suppose that the hot wire (with $\mathrm{T}_{1}=70^{\circ} \mathrm{C}$ ) is presented on a back side temperature $\mathrm{T}_{0}=34^{\circ} \mathrm{C}$. The wire is seen by the camera under a distance of $50 \mathrm{~cm}$. This wire is $1.5 \mathrm{~mm}^{2}$ cross section (diameter $2.5 \mathrm{~mm}$ ): the 
Distance to Size ratio D/d is thus 200:1 (vertical dashed line). In situation of apparent temperature, the systematic error is evaluated to $-5^{\circ} \mathrm{C}$

For a wire covering 1 IFOV (2,1 mrad or 476:1), the thermosignal will take a value appreciably equal to the half sum of thermosignals $L_{0}$ and $L_{1}$. One thus does not measure correctly the value of the thermosignal and the measurement temperature is not correct for a back side temperature of $20^{\circ} \mathrm{C}$ (systematic error of $\left.22^{\circ} \mathrm{C}\right)$.

If the variation in temperature between the wire and the back side is still reduced, one will be able to correctly measure the wire temperature of low diameter. In imagery, this problem does not worry us: it is a problem of measurement and not of imagery. The camera sees the wire very well. Thus, the IFOV is a concept related to the image and does not imply anything on the validity of measurement.

Let us note finally that if the wire is at the same apparent temperature as the bottom. the camera does not see it but it measures very well the temperature of it since the temperatures are spatially identical.

What say the standard? The PRSM of the camera is defined by the high values of the FRF. The standard requires to record the values of $D$ corresponding to the values of FRF of $0,50,0,90,0,95$ and 0,98 . We present the results on table 3 .

Table 3. Systematic error in temperature for a vertical wire at $70^{\circ} \mathrm{C}$ measured on a back side at $20^{\circ} \mathrm{C}$

\begin{tabular}{|c|c|c|c|c|c|}
\hline PRSM & $d / D$ & $\begin{array}{c}\text { Distance to } \\
\text { Size ratio }\end{array}$ & $\begin{array}{l}\text { Number } \\
\text { of IFOV }\end{array}$ & $\Delta \mathrm{N}$ & $\Delta \mathrm{T}$ \\
\hline & (mrad) & & & (Level) & $\left({ }^{\circ} \mathrm{C}\right)$ \\
\hline $50 \%$ & 2.01 & 498 & $(0.96)^{1}$ & -63 & 22 \\
\hline $90 \%$ & 6.04 & 166 & ${ }^{3}{ }^{3}$ & -13 & 4 \\
\hline $95 \%$ & 8.83 & 113 & ${ }^{4.2)^{4}} 4$ & -7 & 2 \\
\hline
\end{tabular}

This results are not relevant if we don't specify the temperature of test. Let us note that uncertainties or the errors in temperature are expressed in ${ }^{\circ} \mathrm{C}$ and not expressed as a percentage of a temperature.

\section{Conclusion}

A fine analysis of the imagery process showed the limits of the Spatial resolution of observation. A quantified study of the spot of diffusion is given and indicates a strong sensitivity with $\mathrm{f} / \mathrm{N}$ of the camera and its spectral band. This limits reduce the exactitude in temperature. We propose a second reading of the signal provided by the observation of a slit presenting a strong thermal contrast. It should be noted that systematic error depend on four parameters : wire temperature, wire orientation, back side temperature and Distance to Size Ratio. Systematic error are presented on a new single graph providing direct access for any of this parameters. It appears more relevant than current French standards 


\section{REFERENCES}

[1] Dominique Pajani. Thermographie. technologies et applications. Techniques de l'ingénieur R 2741

[2] Thermographie infrarouge : caractérisation de l'appareillage. NF A 09-420 et méthodes de caractérisation de l'appareillage. NF A 09-421

[3] NF A 09-400. Thermographie infrarouge. Vocabulaire. Décembre 1991. NF A 09-420.

[4] Dominique Pajani. Résolutions spatiales des caméras thermiques à matrice de détecteurs. Thermogram' 2005.

[5] G. Gaussorgues. la thermographie infrarouge : principes. technologies. applications (4ème édition). pp 160, 164, 211, 